\title{
Design and Implementation of Enterprise the Reimbursement Management System Based on SSH Architecture
}

\author{
Guijun Liu, Chunshan Yang \\ Department of Computer Science and Technolgy, \\ Cheng-Dong College of Northeast Agricultural University \\ Harbin, 150025, China \\ 271110412@qq.com
}

\begin{abstract}
It is based on $\mathrm{c} / \mathrm{s}$ and $\mathrm{b} / \mathrm{s}$ mode for traditional enterprise claims management system, system level architecture is unclear, invasive, and reusability lower defects, and increases the workload and complexity of management. In view of this, uses the MVC pattern and the SSH technology, build tiered architecture, Enterprise claims management system the separation of business logic and control logic, on the basis of design and implementation based on $B / S$ model of enterprise claims management system. Practical application shows that enterprise claims management system framework based on SSH hierarchy clear, reduce the degree of coupling between modules, improved granularity of reuse, enhanced system modules can be reused, flexibility, and maintainability
\end{abstract}

Keywords-component: Struts; Spring ; Hibernate ; reimbursement managemen

\section{INTRODUCTION}

In recent years, based on J2EE open source framework as a substitute for traditional EJB architecture, very rapid development, has a significant impact on software development, is one of the most closely watched Struts, Hibernate and Spring of the three frameworks. Accompanied by the increasing complexity of applications, in order to quickly improve development efficiency, excellent integration framework to accommodate the varied requirements of your applications has become one of the popular direction of software development. Enterprise claims management system provides information for decision-making under the leadership of services. The other hand, Enterprise claims management systems because of the amount involved and complex, involving many posts and personnel, processes cumbersome and varied features, and became one of the IT construction difficulties. Given the Enterprise claims management system focused on both the current software development and difficulties, SSH framework is to resolve J2EE framework an efficient solution to the problem, so, using $\mathrm{SSH}$ integration development enterprise claims management system. Struts+Spring+Hibernate integrated way to formation of a systems development framework, want to be able to complement each other, Struts into full play in the presentation layer and the control layer, Spring in the business layer, Hibernate data persistence layer edge, to improve development efficiency, reduce development effort, reducing the coupling, improve maintainability and scalability goals. The main principle of this article is to look at SSH frameworks, core functions and SSH integration technologies, and technologies applied to the development of enterprise claims management system, reflect the advantages of SSH integration[1-2].

\section{SSH FRAME STRUCTURE MODEL}

Lightweight J2EE development platform takes on J2EE component-based multi-tier application model. By combining the user interface, business logic, and data storage capabilities, and on the basis of logic, and logical classification levels based on application functionality, with clearly defined responsibilities, achieving loosely coupled component architecture. On the technical level, use the Spring when planning a lightweight J2EE application platform, can be applied in the duties would be divided into four layers: the domain model layer, business layer and persistence layer, the presentation layer. Spring connection hub between other layers as the business layer, allows the layer to other layers exposed in a loosely coupled manner between features without having to rely on a specific technology. Other layers are responsible for the application of duties in various directions, to package the feature, clearly defined interfaces between the layers and layers of communication. SHH frame structure model as shown in Figure 1

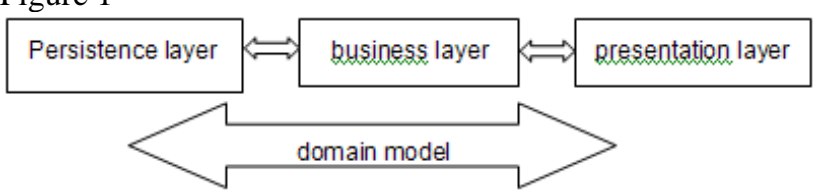

Figure $1 \mathrm{SSH}$ frame structure model

(1) the domain model. In one application, you first need to use a set of objects to express concepts in the real world. The traditional method is to use the DTO (data transfer object) to transform the data in the database for objects in the problem domain at the time of application development requires additional coding. Introduction of model layers, using a set of business objects to achieve impedance matching of the problem domain and the computer field, alternative data transfer object in the system: description of the real world with an object, true reflection of real-world objects (such as orders, products, and so on), you can reduce the additional encoding, easy to understand, reduce code management more difficult. In addition, the business domain model objects to pass data between tiers, and data 
transformation, as carriers of data communication between each layer, reducing the overhead of passing data between systems to maximize, and business objects that represent real-world objects, makes the system at the time of expansion, remodeling, data transfer between parts of the system are not affected[3].

(2) the presentation layer. The presentation layer is part of the user and software interaction. Presentation layer to the user show the functionality of a software system, in response to a user request, and present the results to the user. Thus if the user interface is not efficient, so no matter how excellent the rest, will not help. Mature ways that MVC (model--view-controller) mode of organization presentation layer to separate user interface code (view) and application data and business logic (model), and then use the controller matches the view and the model, data reduction, coupling of data description and application operations, ultimately be answered fast, beautiful interface layer.

(3) the persistence layer. Persistence layer in your application's end, is responsible for datapersistence. Data persistence layer design goal is to provide a high level for the entire project, unity, security, concurrency data persistence mechanisms, the complex business logic and logical separation of data, making the system tightly coupled relationship into loosely coupled relationships, complete the programming of data persistence, and the business logic layer provides the system services. Data lasting layer provides has data access logic, to using programmer avoid manual prepared program access data lasting layer, makes its focused $\mathrm{Yu}$ business logic of development, and to in different of project in the reuse has has achieved, simplified data increased, and by deleting, and check, and modified, features of development process, while and does not lost multilayer structure of natural advantage, inherited continued J2EE special some can telescopic sexual and can extended sexual.

(4) the business layer. From looking at the application layer, the business layer in the middle layer. Business, also known as the service layers. Reasonable construction of the business layer, you can reduce the coupling between levels, increased scalability and flexibility of the business. With Spring IOC container, use the profile management other dependencies, assemble business components at all levels, reducing coupling between levels, implement the plug-in programming; using Spring AOP implementation, focused on enterprise-level services in the system, such as transaction management, log management, and so on, makes the code more concise, enhance reusability, improve development efficiency[4].

In application development, domain object model layer to abstract real-world objects into domains, persistence layer is responsible for the domain object and database mapping, call the business layer data persistence logic. Execute application logic, processes the request and show the results to the user in an appropriate manner.

Struts front control framework simplifies the development of procedures, make the page designers and Java programmers to achieve effective separation, the scalability of the project greatly enhanced, improving development efficiency, reduce maintenance costs.

Spring application as an integrated framework at all levels, application with powerful features and flexibility is ideal as an underlying platform for large-scale software projects.

Hibernate as ORM in the background lightweight persistence layer framework components, a Lightweight Encapsulation of the persistence layer, reducing the complexity of the program, ease of debugging, thereby reducing the burden on the programmer, has a high level of scalability. And open API, Hibernate source code are free to modify and extend the functionality that you need[5].

\section{SSH APPLICATION FRAMEWORK ENTERPRISE CLAIMS MANAGEMENT SYSTEM}

\section{A. Introduction to Enterprise claims management system structure}

Enterprise claims management system consists of the following four modules: claims management (including claims and audits claims), I would like to purchase; I'm going to claim; information centers in four parts.

\section{B. Enterprise claims management system business}

Enterprise claims management system includes four main roles: employee, Manager, the Prime Minister, finance. General staff functions include: adding a claim form, application form will be forwarded to the Manager audit by the system; to have hit back on the application form to modify, view all claims and view individual claims. Department Manager feature in addition to the employee's function, also includes the examination of claims and review the audit claims success. General Manager features include: view a single application form, review the claims and audited more than 5,000 claims for reimbursement. Financial functions include: address by General Manager auditing claims, view a single application form and review the claims. System flow chart as shown in Figure 2

\section{Technical framework, Enterprise claims management system introduction}

In Spring as the core of the system framework of the system, completed data persistence with Hibernate, the presentation layer using Struts2. These frameworks are currently the most popular framework for JavaEE, three frame combinations can guarantee better development effectiveness.

1)Spring applications,Using Spring as a core framework, full use of the advantages of dependency injection, and improve the system of decoupled, improves application scalability, reducing the cost of system reconfiguration.

2)Hibernate application,Hibernate Java persistence layer is an excellent solution that is today's mainstream objectrelational mapping tool. Simplify database access, based on JDBC provides a better package, operations database in an object-oriented manner. 
3) Struts2,Through the Action classes to handle all requests, all JSP pages in the WEB-INF path, prevent users from directly accessing JSP pages can improve the security of the system.

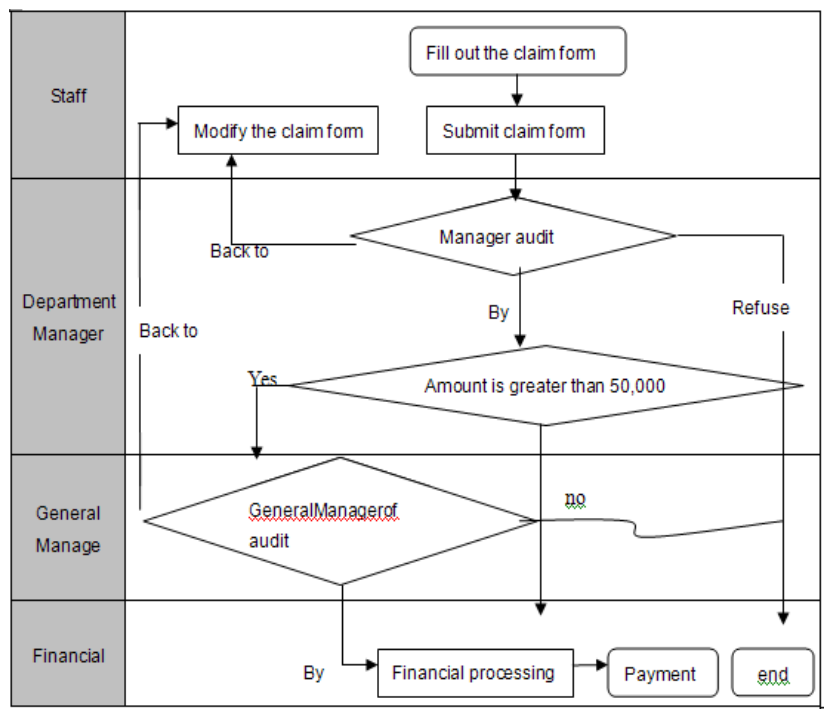

Figure 2 the reimbursement process

D. Database design, Enterprise claims management
system

Under the Enterprise claims management systems business, we analyze what tables are needed. Systems have four roles, correspond to different positions, it is not necessary to record job information data table? Is clearly necessary? A company group structure is made up of multiple sectors, different departments have different functions, so we also need a departments table, record information department name and department managers. The posts table and departments table, now we can design employee information form to record the sectoral career information and basic information for an employee. Information related to an employee table design is completed, and claims related to the table how to design it. First, you need to design claims record, record basic information form, including: informant, fill time, reimbursement of the total amount, claims, claims status and claims audit others. There is a form of basic information, then claims for more information how to record it. So, you also need to design form details table, record claims reimbursements for related details. Records including the claims project, amount and description information. After the above analysis, Enterprise claims management system of database design as shown in Figure 3.

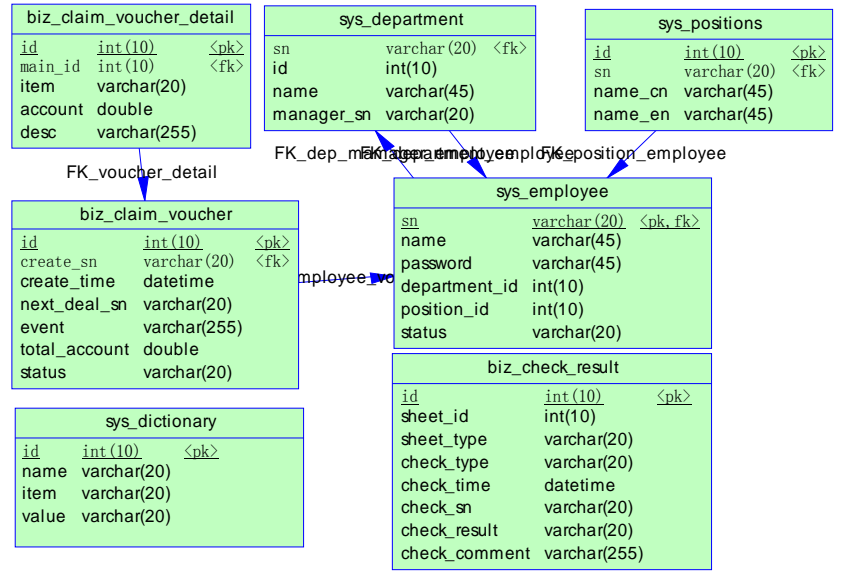

Figure 3 database design

\section{E. Building SSH Enterprise claims management system} framework

1)the use of MyEclipse to create a Web project, name it EnterpriseOA, and then, add Spring, Struts, Hibernate JAR package. Finally, create the include structure, and profiles of the corresponding class. Structure as shown in Figure 4.

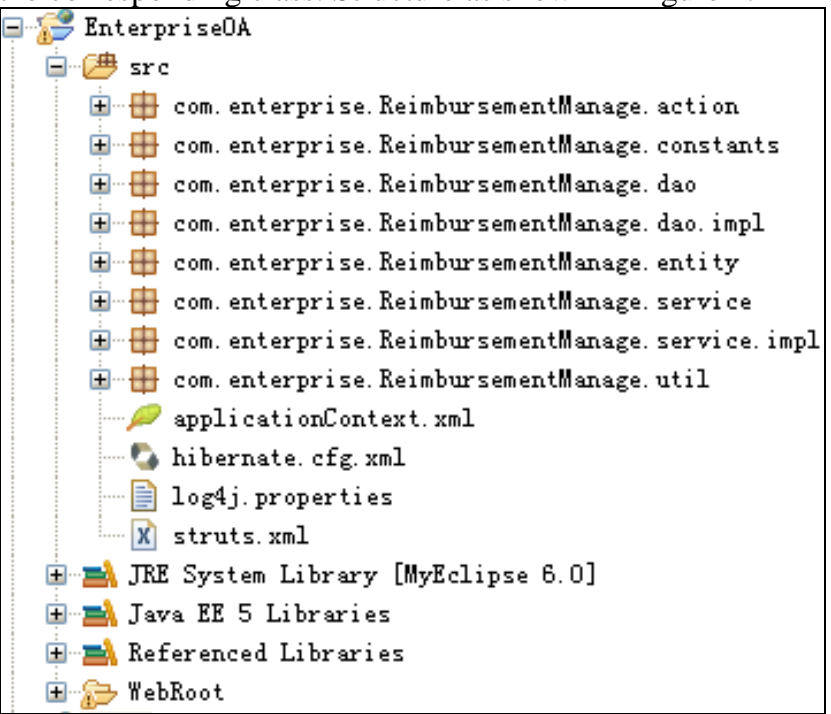

Figure 4 claims management system frame diagram

The basic framework of the project already completed.

2)SSH frameworks enable user login

- Create EmployeeDao interface

package com.enterprise.ReimbursementManage.dao; import java.util.List;

import

com.enterprise.ReimbursementManage.entity.Employee; public interface EmployeeDao \{

public Employee get(String sn);

- Create EmployeeDaoImpl class, inheriting Spring HibernateDaoSupport implements EmployeeDaoImpl interface 
package com.enterprise.ReimbursementManage.dao.impl; import java.util.List;

import

org.springframework.orm.hibernate3.support.HibernateDao Support;

import

com.enterprise.ReimbursementManage.dao.EmployeeDao; import

com.enterprise.ReimbursementManage.entity.Employee;

public class EmployeeDaoImpl extends

HibernateDaoSupport implements EmployeeDao \{

public List $<$ Employee $>$ findEmployee(Employee emp) \{

List $<$ Employee $>$

list

super.getHibernateTemplate().findByExample(emp); return list;

public Employee get(String sn) \{

return (Employee) this.getHibernateTemplate().get(

Employee.class, sn);

\}

\}

Preparation of Spring, Struts2, Hibernate Integration profile.

$<$ ?xml version="1.0" encoding="UTF-8"?>

$<$ web-app version=" $2.5 "$

xmlns="http://java.sun.com/xml/ns/javaee"

xmlns:xsi="http://www.w3.org/2001/XMLSchema-

instance"

xsi:schemaLocation="http://java.sun.com/xml/ns/javaee

http://java.sun.com/xml/ns/javaee/web-app_2_5.xsd">

$<$ context-param $>$

$<$ param-name $>$ contextConfigLocation $<$ /param-name $>$

$<$ param-value $>$ classpath:applicationContext.xml $<$ param-

value $>$

$</$ context-param $>$

$<$ listener $>$

$<$ listenerclass $>$ org.springframework.web.context.ContextLo aderListener $<$ /listener-class $>$
$</$ listener $>$

$</$ web-app $>$

\section{CONCLUSION}

This article highlighted Struts+Spring+Hibernate framework in the design and application of enterprise management in the reimbursement system. In a traditional J2EE application, EJB has been occupying the leading position, but it needs a huge container, popularly known as "heavy-weight container". Because EJB has exposed flaws and complexities, "lightweight container" as the core development of combination of schema Struts+Spring+Hibernate solved the problem. SSH schema presentation layer with Struts, the business layer with Spring, Hibernate for persistence, is the development of a more simple, flexible, system maintenance is also more convenient, is senior developers are more concerned about the program the business logic implementation, reducing the underlying framework of design considerations, improving development efficiency.

\section{REFERENCES}

[1] Zi-yao Wang, Jun-jie Nan and Zi-hui Duan “SOA Core technology and applications ”,Bei-jing, Publishing House of Electronics Industry,2008,PP.34-39

[2] Guo Guangjun, Xie Dong, Chen Li, teaching support system based on SSH integration framework design and implementation. in computer engineering and design, 2009, PP.30-32

[3] Liang $\mathrm{Bi}$, SSH integration technology research and application of the framework, Journal of Xian Academy of Arts and Sciences: Science, 2011, PP.17-19

[4] Deng Lujuan, Sun Yikun, diao Harbour, based on student result management system management system design and realization of Struts+Spring+Hibernate, 2010, PP.12-15

[5] Sun Zuoren, Li Hongwei, Chen Yu and. Green based on SSH University evaluation support system . Computer applications and software, 2009 , PP. 102-104. 